\title{
Upsurge of malaria transmission after indoor residual spraying withdrawal in Atacora region in Benin, West Africa
}

\author{
Rock Yves Aïkpon ${ }^{1,2,3^{*}}$, Gil Padonou ${ }^{2,4}$, Fortuné Dagnon ${ }^{5}$, Razaki Ossè ${ }^{2,6}$, Aurore Ogouyemi Hounto 7,8 , \\ Filémon Tokponon ${ }^{8}$, Gorgias Aïkpon ${ }^{4}$, Laurent Lyikirenga ${ }^{9}$ and Martin Akogbéto ${ }^{1}$
}

\begin{abstract}
Background: In Benin, malaria vector control mostly relies on long-lasting, insecticidal-treated bed nets (LLINs) and indoor residual spraying (IRS) operations. From 2011 to 2016, an IRS programme has been implemented in Atacora region. However, in 2017 the programme was withdrawn from two other regions in the northern part of the country, with hopes that gains would be relatively sustained because of the seasonality of malaria transmission. What would be the vulnerability of populations to malaria after the withdrawal of IRS?

Methods: Monthly mosquito collections were performed through human landing captures (HLCs) for 24 months (from January to December 2016 during the last IRS campaign, and from January to December 2018, 2 years after the withdrawal of IRS). Vector mosquitoes biting density was sampled by HLC and was tested for presence of Plasmodium falciparum sporozoites. The carcass of these mosquitoes (abdomens, wing, legs) were subjected to molecular species identification using polymerase chain reaction (PCR) assays.
\end{abstract}

Results: It is noticed a drastic increase ( 3 times higher) of vector abundance after the withdrawal of IRS. Mosquito biting rates in the 3 survey districts increased significantly after IRS was withdrawn. In 2018, after IRS cessation a significant increase of entomological inoculation rate was recorded, where each inhabitant received an average of 94.9 infected bites/year to 129.21 infected bites/year against an average of 17.15 infected bites/year to 24.82 infected bites/year in 2016.

Conclusion: It is obvious that the withdrawal of IRS confers a vulnerability of the population with regard to the malaria transmission. Robust monitoring is needed to better understand when and where IRS should be most adequate, or can be safely withdrawn. In case of withdrawal, adapted accompanying measures should be proposed according to the context not only to maintain the gains capitalized with IRS, but also to avoid any rebound of transmission.

Keywords: Indoor residual spraying, Withdrawal, Malaria, Upsurge, Benin

*Correspondence: rockypremier@yahoo.fr

${ }^{1}$ Centre de Recherche Entomologique de Cotonou (CREC), Cotonou, Benin

Full list of author information is available at the end of the article

\section{Background}

Malaria remains a serious threat to development, with an estimated 215 million cases and 438,000 deaths in 2015 , of which $88 \%$ of cases and $90 \%$ of deaths occurred in sub-Saharan Africa [1]. Vector control is a fundamental component in malaria prevention strategies and has contributed to a significant decrease in malaria worldwide [2-4]. It relies primarily on two complementary

c) The Author(s) 2020. This article is licensed under a Creative Commons Attribution 4.0 International License, which permits use, sharing, adaptation, distribution and reproduction in any medium or format, as long as you give appropriate credit to the original author(s) and the source, provide a link to the Creative Commons licence, and indicate if changes were made. The images or other third party material in this article are included in the article's Creative Commons licence, unless indicated otherwise in a credit line to the material. If material is not included in the article's Creative Commons licence and your intended use is not permitted by statutory regulation or exceeds the permitted use, you will need to obtain permission directly from the copyright holder. To view a copy of this licence, visit http://creativeco mmons.org/licenses/by/4.0/. The Creative Commons Public Domain Dedication waiver (http://creativecommons.org/publicdomain/ zero/1.0/) applies to the data made available in this article, unless otherwise stated in a credit line to the data. 
tools, including long-lasting insecticidal nets (LLINs) and indoor residual spraying (IRS) [5-7]. LLINs have contributed greatly to reducing malaria morbidity and mortality in many endemic areas, and the World Health Organization (WHO) recommends universal coverage of at-risk populations $[8,9]$. IRS has also been shown to be highly effective, particularly where mosquito vectors feed and rest indoors, and in situations of seasonal malaria transmission [10-12]. However, IRS requires more resources and logistics to implement than the distribution of LLINs. Currently, less than $10 \%$ of the population at risk in sub-Saharan Africa is protected by IRS $[1,13,14]$.

Malaria is endemic in Benin, with 1.5 million cases reported annually among a national population of 11.1 million. However, progress in reducing malaria burden has been slowest. In Benin, malaria vector control relies on the mass distribution of LLINs and IRS operations. From 2011 to 2016, an IRS programme was implemented with funding from the US President's Malaria Initiative (PMI), in north Benin and targeted all houses in the Atacora region, an epidemic-prone area in Benin. During those 6 years of IRS implementation, two insecticides of two different classes were used in rotation: bendiocarb (a carbamate) and pirimiphos methyl (an organophosphate), due to the emergence and expansion of resistance of Anopheles vectors to insecticides, especially pyrethroids [15-22]. After 6 years of implementation (2011-2016), IRS showed a significant reduction in malaria transmission in Atacora region [23, 24]. However in 2017, the IRS programme was moved to two other regions (Donga and Alibori) in the north, with hopes that gains would relatively be sustained because of the seasonality of malaria transmission.

Few entomological studies investigated vector control withdrawal and its implications on subsequent malaria transmission trends. The entomological indicators of malaria transmission such as vector abundance, human biting rate (HBR) and entomological inoculation rate (EIR) are parameters commonly used to assess the impact of vector control interventions and the intensity of malaria transmission. In this study, it is proceeded with the entomological monitoring in Atacora region 2 years after the withdrawal of IRS. The primary objective was to assess trends in entomological indicators of malaria transmission during IRS implementation, and after its withdrawal.

\section{Methods}

\section{Intervention areas}

The study was carried out in the department of Atacora (which is composed in total of 9 districts: Kouandé, Natitingou, Toukountouna, Kouandé, Boukoumbé, Pehunco, Cobly, Tanguiéta, and Toukountouna) located in the northwest of Benin. The study includes three districts out of nine: Kouandé, Natitingou and Toukountouna (Fig. 1). These three districts cover about $6384 \mathrm{sq}$ $\mathrm{km}$ and had an estimated population of 326,868 in 2015 . Atacora region has a sub-equatorial type climate with one dry season (December-May) and one rainy season (June-November). The annual mean rainfall is $1300 \mathrm{~mm}$ and the monthly mean temperature varies between 22 and $34{ }^{\circ} \mathrm{C}$. The main economic activity is agriculture and it is characterized by the production of cotton and millet where various classes of pesticides are used for pest control.

Data were collected in these districts in 2016 (during the last IRS campaign) and in 2018 (2 years after the withdrawal of IRS). The same entomological indicators of malaria transmission were compared between 2016 and 2018.

\section{IRS campaigns (2011-2016)}

From 2011 to 2016, a yearly IRS round was implemented in the Atacora region at the beginning of the rainy season and targeted all eligible households. Each round covered over $90 \%$ of the households in the target districts (Table 1). More than 500,000 inhabitants were protected by IRS operation each year in Atacora region. The first product chosen by the National Malaria Control Programme (NMCP) to implement IRS in Atacora was carbamate, bendiocarb [25]. The formulation was $80 \%$ Wettable Powder (WP). The IRS operation was performed by volunteers chosen from the local community and trained by the PMI IRS partner.

Anopheles gambiae resistance to bendiocarb was observed after 2 years of IRS implementation using bendiocarb in Atacora region in Benin [26]. In order to lower the bendiocarb selection pressure, bendiocarb was replaced in 2013 by pirimiphos methyl (Actellic 300CS) an organophosphate that was used from 2013 to 2016. The residual effect of bendiocarb and pirimiphos methyl (> $80 \%$ mortality in cone bioassays) lasted four months in field conditions.

\section{Entomology surveys}

In each district, monthly mosquito collections were performed through HLCs on two consecutive nights from 19.00 to $06.00 \mathrm{~h}$, for 24 months (from January to December 2016 during the last IRS campaign and from January to December 20182 years after the withdrawal of IRS).

The collection was carried out with a mouth aspirator by volunteers who had given their consent. The collectors were given anti-malarial prophylaxis as a prevention against malaria. During the course of the study, all mosquito collectors were monitored for malaria 


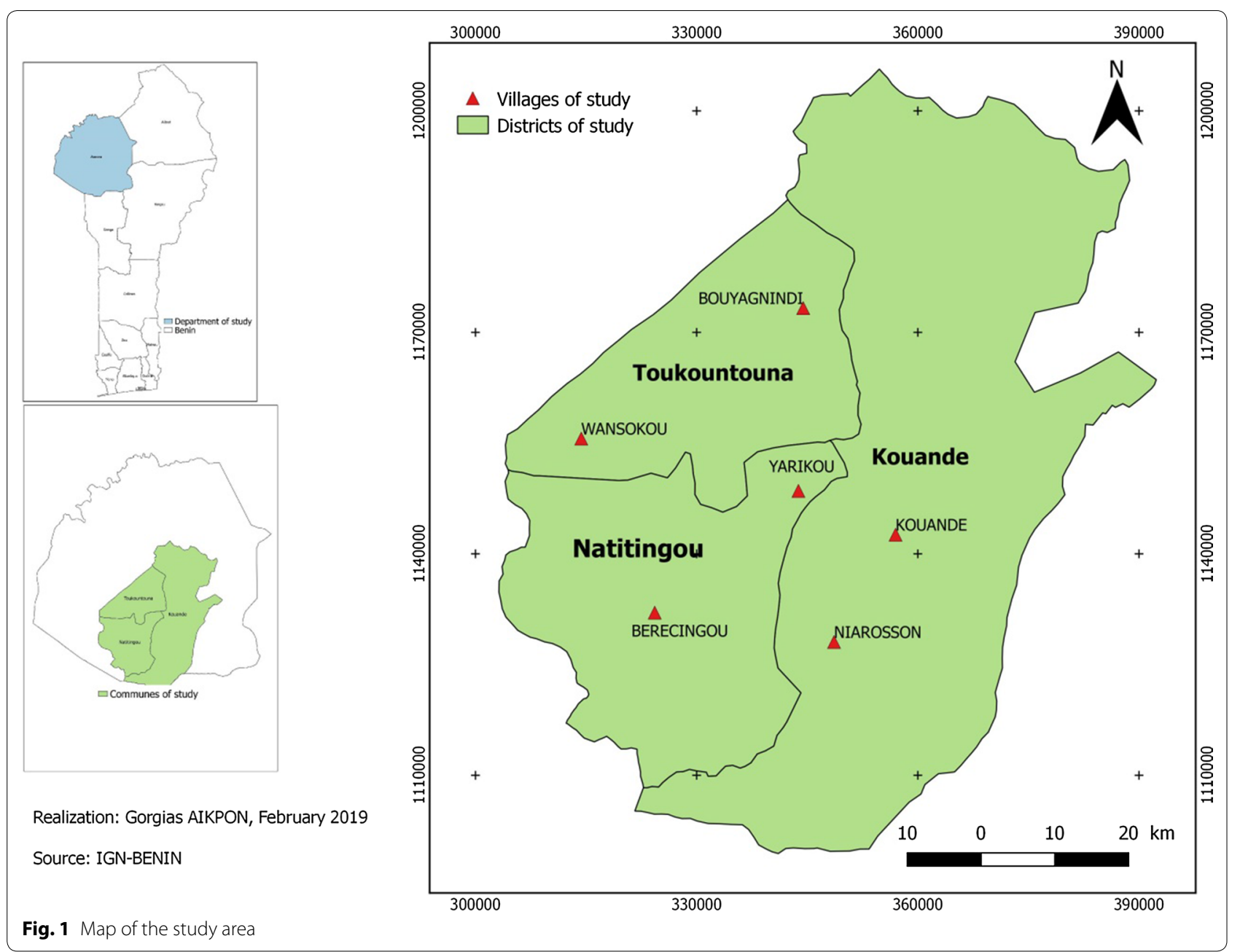

Table 1 Details of indoor residual spraying of insecticide in Atacora department

\begin{tabular}{|c|c|c|c|c|c|c|c|}
\hline Year & Department & $\begin{array}{l}\text { Number } \\
\text { of districts } \\
\text { covered }\end{array}$ & Period of spraying & Class of insecticide used & $\begin{array}{l}\text { Number } \\
\text { of structures } \\
\text { treated }\end{array}$ & Coverage (\%) & $\begin{array}{l}\text { Population } \\
\text { protected }\end{array}$ \\
\hline 2011 & Atacora & 9 & April-May & Carbamate (Bendiocarb) & 170,000 & 93 & 500,000 \\
\hline 2012 & Atacora & 9 & April-May & Carbamate (Bendiocarb) & 210,380 & 94.8 & 652,777 \\
\hline 2013 & Atacora & 9 & April-May & Organophosphate (Pirimiphos methyl-EC) & 228,915 & 95.7 & 694,729 \\
\hline 2014 & Atacora & 9 & April-May & Organophosphate (Pirimiphos methyl-CS) & 254,072 & 95.5 & 789,883 \\
\hline 2015 & Atacora & 9 & April-May & Organophosphate (Pirimiphos methyl-CS) & 252,706 & 93.5 & 802,597 \\
\hline 2016 & Atacora & 9 & April-May & Organophosphate (Pirimiphos methyl-CS) & 269,179 & 90.8 & 858,113 \\
\hline
\end{tabular}

symptoms, which triggered an immediate parasitological test followed by an anti-malarial treatment when necessary. Two houses were selected at random per district for the HLC. In each house, one collector was positioned indoor and another one outdoor.

\section{Laboratory processing}

Collected mosquitoes were identified at species level based on morphological criteria according to established taxonomic keys [27, 28]. Female vector mosquitoes were tested to determine the percentage of mosquitoes that were positive for sporozoites based on an ELISA test [29]. 
Moreover, the carcasses of these mosquitoes (abdomens, wings and legs) were subjected to molecular species identification using polymerase chain reaction (PCR) assays [30].

\section{Statistical analyses}

Data were collected using standardized data collection forms and entered using Microsoft Access. Vector density during IRS campaign period and after its withdrawal was assessed. The primary metrics such as human biting rate (number of bites/man/night) (HBR), sporozoïte rate (Is), EIR and blood feeding rate (number engorged divided by total collected) were estimated with confidence intervals at $95 \%$. These rates were then compared during the IRS period (2016) and 2 years after its withdrawal using the Chi square test. Significant differences were those with a p-value of $<0.05$.

\section{Results}

\section{Change in Anopheles species abundance}

A total of 1540 adult female Anopheles mosquitoes belonging to 4 different species were collected with a predominance of An. gambiae over the 2 years (2016 and 2018). A drastic increase ( 3 times higher) in vector abundance was noticed after the withdrawal of IRS (Fig. 2). PCR results showed the presence of An. gambiae and Anopheles coluzzii and Anopheles arabiensis, as sibling species of Anopheles gambiae complex. In addition, all mosquito specimens of the Anopheles funestus group were found to be Anopheles funestus sensu stricto (s.s.).
The same vector species were observed before and after IRS.

\section{Comparison of HBRs during the IRS implementation versus $\mathrm{HBRs}$ following IRS withdrawal}

Figure 2 compares the dynamic of HBRs during IRS intervention (2016), with similar estimates following IRS withdrawal (2018). Mosquito biting rates in the three survey districts increased significantly after IRS was withdrawn (Fig. 3). In Toukountouna district, the average HBR was 5.625 bites/human/month during the IRS campaign against 35.625 after IRS withdrawal (Table 2). The same observation was made in Natitingou district where the average HBR was 3.75 bites/human/month during the IRS campaign against 11.25 after IRS withdrawal. In Kouandé district the average HBR was 1.875 bites/ human/month during the IRS campaign against 35.625 after IRS withdrawal.

Figure 4 shows vector exophagic behaviour during the IRS intervention period and after its withdrawal. A significant decrease of exophagy rate was observed after IRS withdrawal. This means that vectors bit more indoors after the IRS withdrawal and sometimes four times more than before (during IRS).

\section{Shift in EIR after IRS withdrawal}

During the IRS intervention period in 2016, 393 heads and thoraxes of female vector mosquitoes were tested using ELISA CSP to identify the presence of sporozoites. Thirty-one $(7.88 \%)$ tested positive for Plasmodium

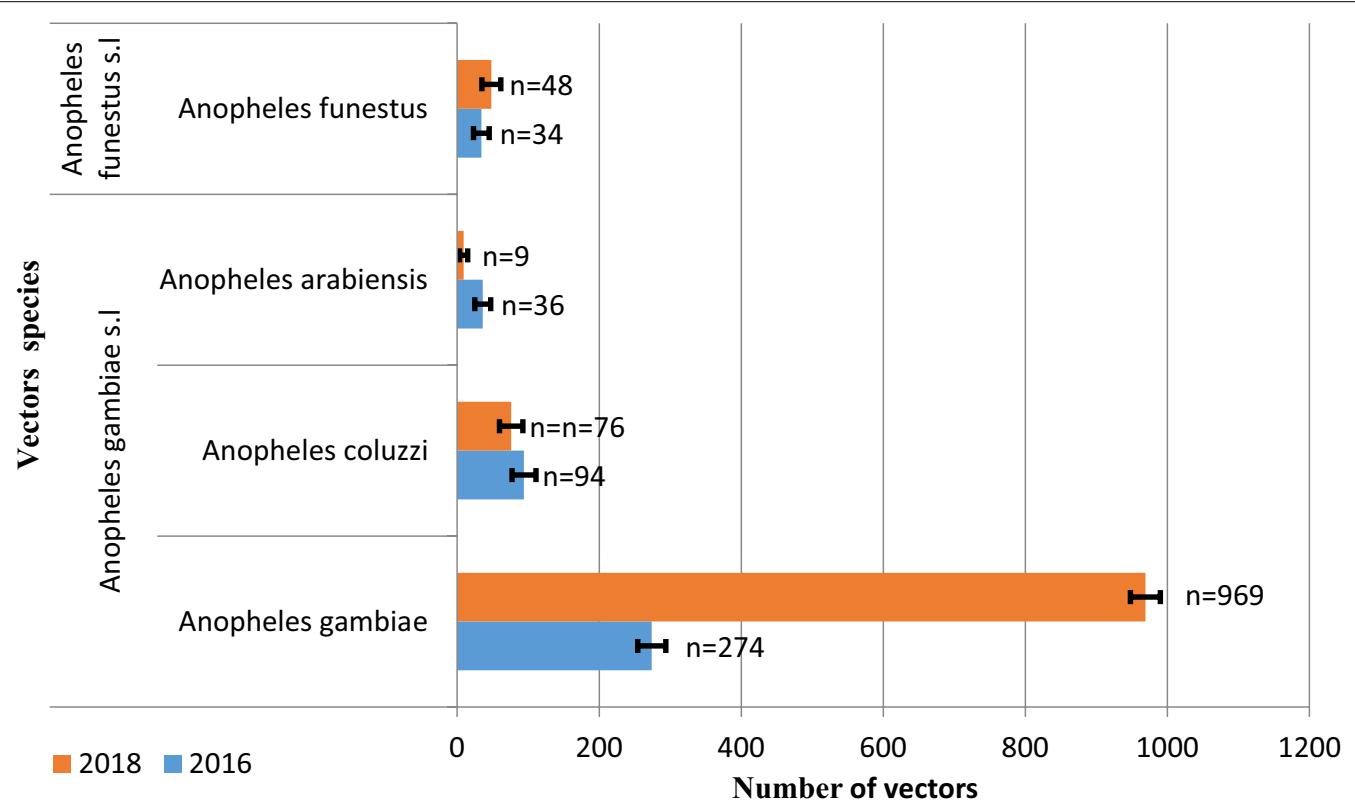

Fig. 2 Vectors species composition and abundance during and after IRS 

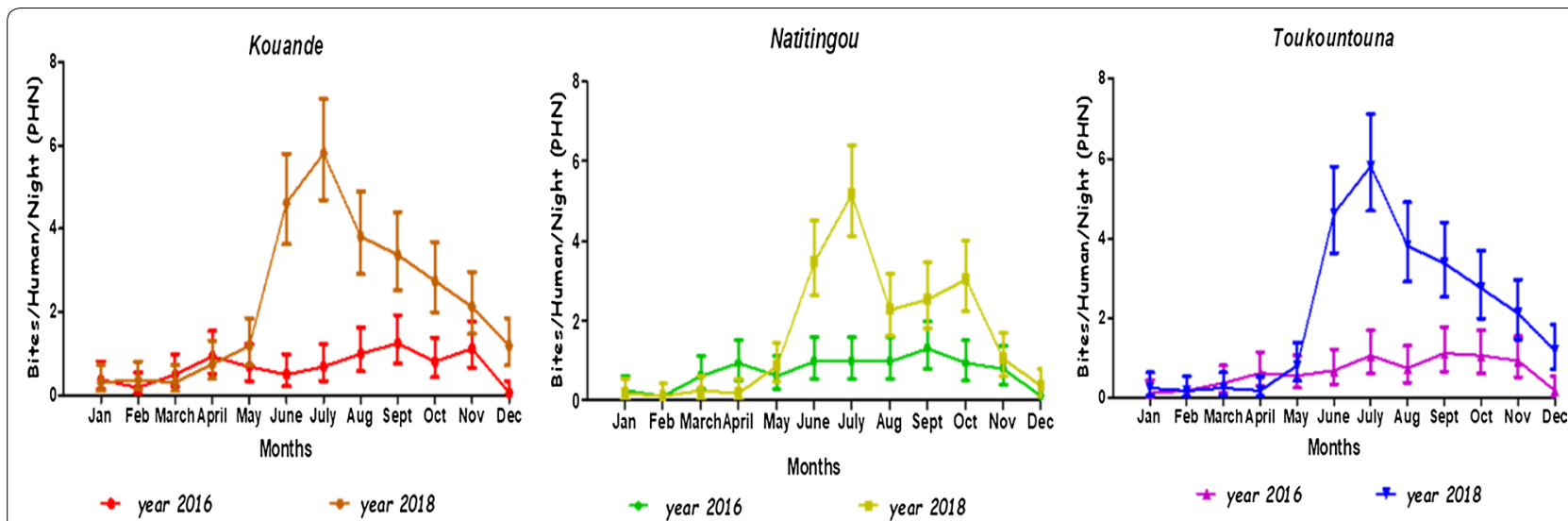

Fig. 3 Dynamic of HBR observed during IRS campaign and following IRS withdrawal

falciparum sporozoite antigen. In 2018, after the IRS withdrawal, a total of 1147 heads and thoraxes of female vector mosquitoes were tested using ELISA CSP, on which 169 (14.73\%) tested positive for P. falciparum sporozoite antigen. The sporozoite index almost doubled from 2016 to 2018.

On the other hand, during the IRS intervention period, each inhabitant of the three districts received an average of 17.15 infected bites/year to 24.82 infected bites/year. In 2018, after IRS cessation, record showed a significant increase of EIR where each inhabitant would receive an average of 94.9 infected bites/year to 129.21 infected bites/year (Table 2).

\section{Discussion}

In Benin, the benefits of IRS were clear, as evidenced by the dramatic decrease in entomological malaria transmission indicators since its implementation in Atacora region in 2011 [23, 24]. However, after the 2016 IRS campaign, the programme was withdrawn from Atacora region and moved by NMCP to two other regions (Donga and Alibori) in the north, with hopes that gains would be relatively sustained because of the seasonality of malaria transmission. What would be the vulnerability of populations to malaria after the withdrawal of IRS in the region of Atacora? Following the withdrawal of IRS, a universal LLIN distribution campaign was conducted in August 2017 with hopes that gains achieved by IRS would be maintained.

A previous study from Benin that compared IRS combined with LLINs versus either intervention alone has shown that adding IRS to the use of LLINs appears to be most effective [31] and similar results are also reported in Gambia [32].

In this study, the upsurge of malaria occurred despite universal LLIN distribution following IRS withdrawal.
The results raise several questions. The first one is whether LLINs were used properly in this population. Indeed, the effectiveness of LLINs depends on certain parameters, such as high coverage, proper use and 'net culture' in a community. Further investigations of LLIN use in this population are required. Another aspect that may limit the effectiveness of LLINs is the high resistance of malaria vectors to pyrethroid that has been reported in Benin and other parts of Africa [33]. Shifts observed in the relative abundance of malaria vector species, without forgetting probable changes in vector behaviour, may increase exposure risk [34].

WHO recommended IRS as a key intervention for the reduction and elimination of malaria in Africa, and IRS coverage, therefore, increased in the last decade in Africa. However, many programmes have to cope with the growing resistance of malaria vectors to insecticides on the one hand and resource constraints on the other. This situation has led many countries to withdraw IRS a few years after its implementation, or to reduce the intervention coverage rate. As a result, a reduction to more than half the number of sprayed houses across 18 countries in Africa, supported by the PMI in 2015, was reported compared to 2008 [35].

Very few studies have assessed the impact of IRS withdrawal on malaria transmission, as the first cases of withdrawal are recent in Africa. This study imputes the upsurge of malaria to the withdrawal of IRS from the study area. A similar situation was reported in Cape Verde, Sri Lanka and Turkey where reduction in IRS coverage was linked to malaria resurgence [36, 37]. An increase in malaria prevalence was also reported following the discontinuation of IRS after 4 years despite distribution of LLINs in Tanzania [35]. However, contrary to all these reported cases, a previous pilot study conducted in Tanzania in 1959 showed that the 


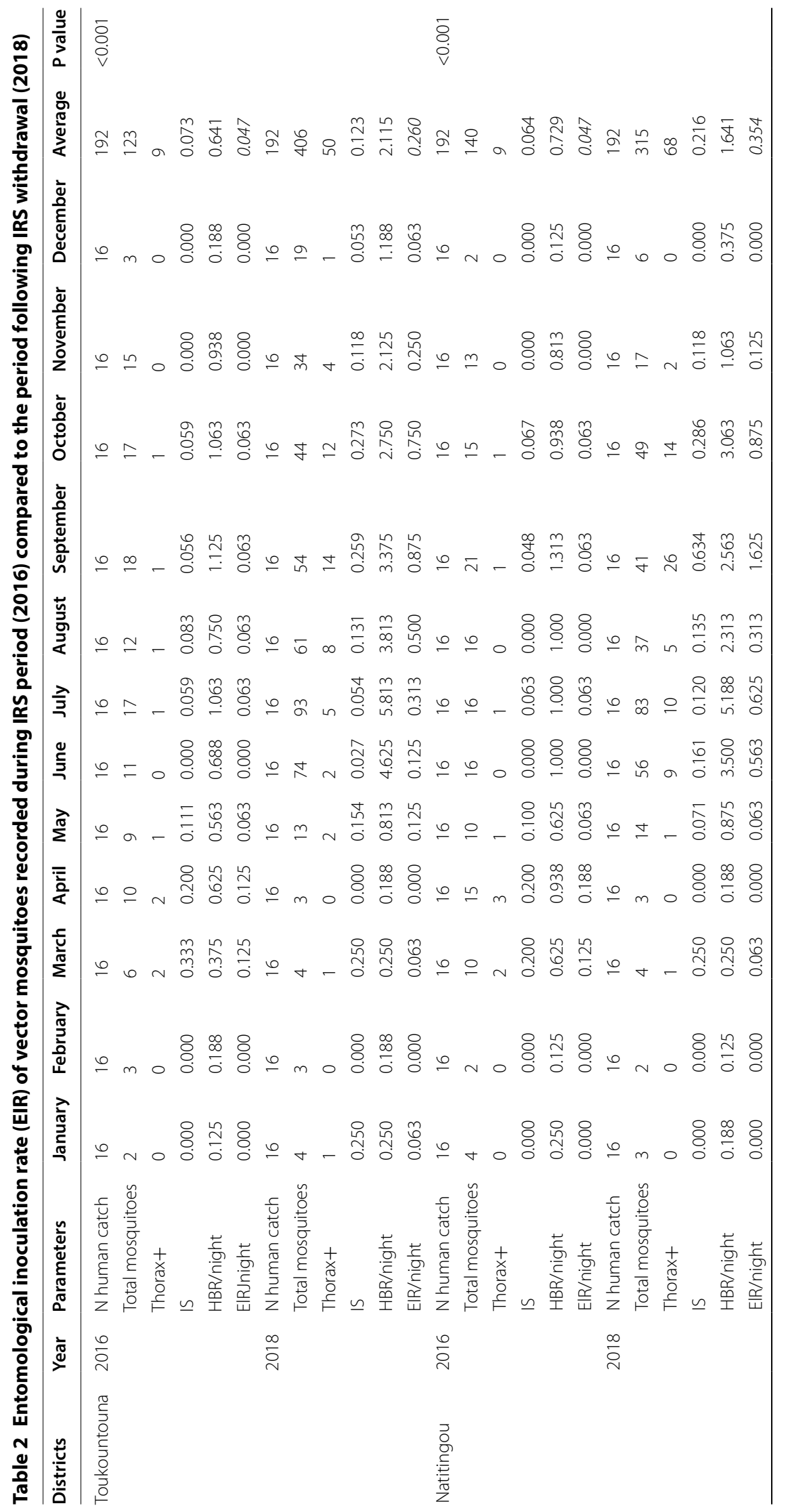




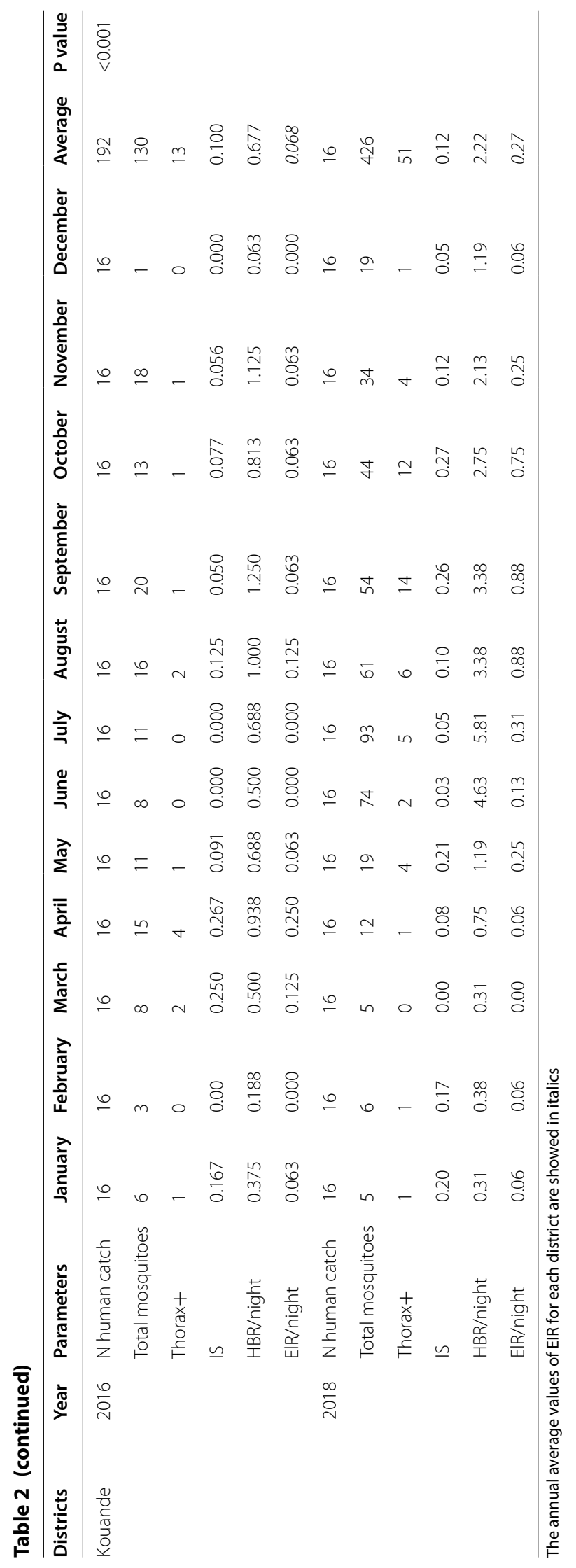



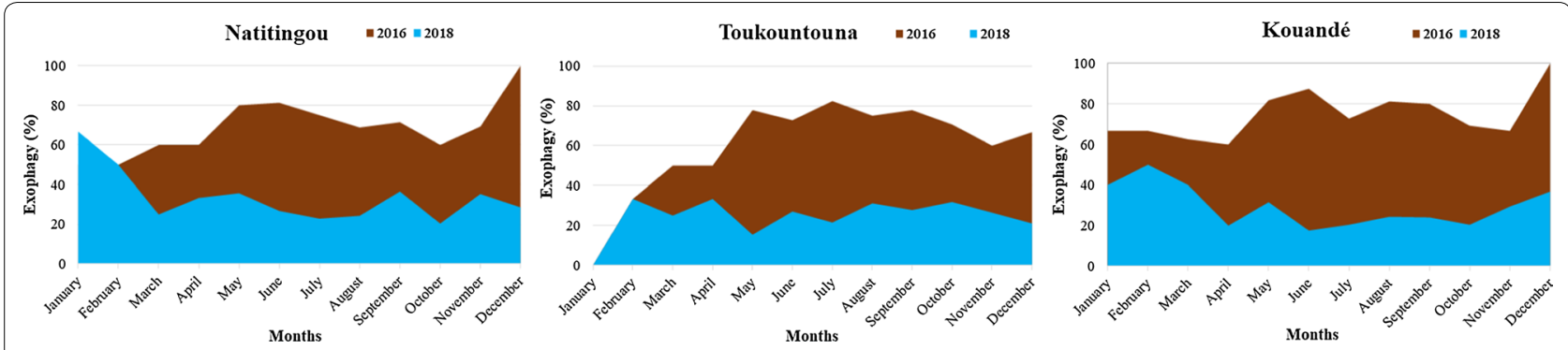

Fig. 4 Vectors exophagic behavior during the IRS intervention period and after its withdrawal

resurgence of malaria occurred many years later after IRS cessation [37, 38].

It is important to mention some of the limitations of this study namely, the fact that parameters other than the cessation of IRS could contribute to the upsurge of malaria in the study area.

\section{Conclusion}

It is obvious that the withdrawal of IRS lends a vulnerability to the population with regard to malaria transmission. In the context where availability of funds is insufficient to ensure broad and sustainable coverage of IRS, many African countries are facing difficult decisions on how to maintain IRS as a key vector control strategy. Therefore, robust monitoring is needed to better understand when and where IRS should be most adequate, or can be safely withdrawn. In case of withdrawal, adapted accompanying measures should be proposed according to the context in order not only to maintain gains capitalized with IRS, but also to avoid any rebound of transmission.

\section{Abbreviations}

HLC: human landing catche; HBR: human biting rate; ELISA: enzyme-linked immunosorbent assay; PCR: polymerase chain reaction; SI: sporozoite index: EIR: entomological inoculation rate; CSP: circumsporozoite protein.

\section{Acknowledgements}

This work was financially supported by PMI (President's Malaria Initiative) through USAID. We thank the team of CREC for their technical assistance during field work and the laboratory. We also thank the people of Atacora for their collaboration.

\section{Authors' contributions}

$R A, G P, F D, R O, A O H, F T, G A, L L$ and MA designed the study. RA, GP, FD, RO and GA performed the field activities. RA and RO carried out the laboratory's activities. RA drafted the manuscript and analysed the data. MA, LL, AOH, FT and GA critically revised the manuscript for intellectual content. All authors read and approved the final manuscript.

\section{Funding}

This work has been financially supported by Centre de Recherche Entomologique de Cotonou (CREC).

\section{Availability of data and materials}

The data supporting the conclusions of this article are included within the article. The raw data used in this study are available from the corresponding author upon reasonable request.

\section{Ethics approval and consent to participate}

This study was received the ethical approval by the Institutional Ethics Committee of CREC (IECC). The mosquito collectors gave their consent before participate to this study. They were checked up by treatment malaria after mosquito collection and were vaccinated against yellow fever.

\section{Consent for publication}

Not applicable.

\section{Competing interests}

The authors declare that they have no competing interests.

\section{Author details}

${ }^{1}$ Centre de Recherche Entomologique de Cotonou (CREC), Cotonou, Benin. ${ }^{2}$ Ecole Normale Supérieure de Natitingou (ENS), Natitingou, Benin. ${ }^{3}$ Université Nationale des Sciences, Technologies, Ingénierie et Mathématiques (UNSTIM), Abomey, Benin. ${ }^{4}$ Université d'Abomey-Calavi, Cotonou, Benin. ${ }^{5}$ US President's Malaria Initiative, US Agency for International Development, Cotonou, Benin. ${ }^{6}$ Université Nationale d'Agriculture (UNA), Ketou, Benin. ${ }^{7}$ Faculté des Sciences de la Santé (FSS), Cotonou, Benin. ${ }^{8}$ Programme National de Lutte Contre le Paludisme, Cotonou, Benin. ${ }^{9}$ Abt Associate, VectorLink, Cotonou, Benin.

Received: 9 October 2019 Accepted: 21 December 2019

Published online: 03 January 2020

\section{References}

1. WHO. World malaria report 2015. Geneva: World Health Organization; 2015.

2. WHO. global plan for insecticide resistance management. Geneva: World Health Organization; 2012.

3. Shargie EB, Ngondi J, Graves PM, Getachew A, Hwang J, Gebre T, et al. Rapid increase in ownership and use of long-lasting insecticidal nets and decrease in prevalence of malaria in three regional States of Ethiopia (2006-2007). J Trop Med. 2010;2010:e750978.

4. Otten M, Aregawi M, Were W, Karema C, Medin A, Bekele W, et al. Initial evidence of reduction of malaria cases and deaths in Rwanda and Ethiopia due to rapid scale-up of malaria prevention and treat ment. Malar J. 2009;8:14.

5. Chavasse DC, Yap HH, WHO. Division of Control of Tropical Diseases. Chemical methods for the control of vectors and pests of public health importance. Geneva: World Health Organization; 1997.

6. Curtis CF, Mnzava AE, Misra S, Rowland M. Malaria control: bednets or spraying? Summary of the presentations and the discussion. Trans R Soc Trop Med Hyg. 1999;93:460. 
7. Okumu FO, Moore SJ. Combining indoor residual spraying and insecticide treated nets for malaria control in Africa: a review of possible outcomes and an outline of suggestions for the future. Malar J. 2011;10:208

8. Lengeler C. Insecticide-treated bed nets and curtains for preventing malaria. Cochrane Database Syst Rev. 2004;2:CD000363.

9. Lim SS, Fullman N, Stokes A, Ravishankar N, Masiye F, Murray CJ, et al. Net benefits: a multicountry analysis of observational data examining associations between insecticide-treated mosquito nets and health outcomes. PLoS Med. 2011:8:e1001091.

10. Schiff C. Integrated approach to malaria control. Clin Microbiol Rev. 2002;15:278-93.

11. Lengeler C, Sharp B. Indoor residual spraying and insecticide-treated nets. In: Murphy C, Ringheim K, Woldehanna S, Volmink J, editors. Reducing malaria's burden: evidence of effectiveness for decision makers. Washington, DC: Global Health Council; 2003. p. 17-24.

12. Roberts D, Curtis C, Tren R, Sharp B, Shiff C, Bate R. Malaria control and public health. Emerg Infect Dis. 2004;10:1170-1.

13. WHO. Indoor residual spraying: use of indoor residual spraying for scaling up global malaria control and elimination. Geneva: World Health Organization; 2006.

14. West PA, Protopopoff N, Wright A, Kivaju Z, Tigererwa R, Mosha FW, et al. Enhanced protection against malaria by indoor residual spraying in addition to insecticide treated nets: is it dependent on transmission intensity or net usage? PLoS ONE. 2015;10:e0115661.

15. Chandre F, Manguin S, Brengues C, Dossou Yovo J, Darriet F, Diabate A, et al. Current distribution of pyrethroid resistance gene $(k d r)$ in Anopheles gambiae complex from West Africa and further evidence for reproductive isolation of Mopti form. Parassitologia. 1999;41:319-22.

16. Hargreaves K, Koekemoer LL, Brooke BD, Hunt RH, Mthembu J, Coetzee M. Anopheles funestus resistant to pyrethroid insecticides in South Africa. Med Vet Entomol. 2000;14:181-9.

17. Etang J, Manga L, Chandre F, Guillet P, Fondjo E, Mimpfoundi R, et al. Insecticide susceptibility status of Anopheles gambiae s.l. (Diptera:Culicidae) in the Republic of Cameroon. J Med Entomol. 2003;40:491-7.

18. Corbel V, N'Guessan R, Brengues C, Chandre F, Djogbenou L, Martin $\mathrm{T}$, et al. Multiple insecticide resistance mechanisms in Anopheles gambiae and Culex quinquefasciatus from Benin, West Africa. Acta Trop. 2007;101:207-16

19. Yadouleton A, Asidi A, Djouaka R, Braïma J, Agossou C, Akogbeto M. Development of vegetable farming: a cause of the emergence of insecticide resistance in populations of Anopheles gambiae in urban areas of Benin. Malar J. 2009;8:103.

20. Djogbenou L, Pasteur N, Bio-Bangana S, Baldet T, Irish SR, Akogbeto M, et al. Malaria vectors in the Republic of Benin: distribution of species and molecular forms of the Anopheles gambiae complex. Acta Trop. 2010:114:116-22.

21. Djegbe I, Boussari O, Sidick A, Martin T, Ranson H, Chandre F, et al. Dynamics of insecticide resistance in malaria vectors in Benin: first evidence of the presence of L1014S kdr mutation in Anopheles gambiae from West Africa. Malar J. 2011;10:261.

22. Sovi A, Djegbe I, Soumanou L, Tokponnon F, Gnanguenon V, Azondekon $\mathrm{R}$, et al. Microdistribution of the resistance of malaria vectors to deltamethrin in the region of Plateau (southeastern Benin) in preparation for an assessment of the impact of resistance on the effectiveness of long-lasting insecticidal nets (LLINs). BMC Infect Dis. 2014;14:103.

23. Akogbéto MC, Aikpon R, Azondekon R, Padonou G, Osse R, Agossa FR, et al. Six years of experience in entomological surveillance of indoor residual spraying against malaria transmission in Benin: lessons learned, challenges and outlooks. Malar J. 2015;14:242.

24. Aïkpon R, Sèzonlin M, Tokponon F, Okè M, Oussou O, Oké-Agbo F, et al. Good performances but short lasting efficacy of Actellic 50 EC Indoor Residual Spraying (IRS) on malaria transmission in Benin, West Africa. Parasit Vectors. 2014;7:256.

25. Akogbéto MC, Padonou GG, Gbénou D, Irish S, Yadouleton A. Bendiocarb, a potential alternative against pyrethroid resistant Anopheles gambiae in Benin, West Africa. Malar J. 2010:9:204.

26. Aïkpon R, Agossa F, Ossè R, Oussou O, Aïzoun N, Oké-Agbo F, Akogbéto M. Bendiocarb resistance in Anopheles gambiae s.l. populations from Atacora department in Benin, West Africa: a threat for malaria vector control. Parasit Vectors. 2013;6:192.

27. Gillies MT, De Meillon D. The Anophelinae of Africa South of the Sahara. Publ S Afr Inst Med Res. 1968;54:343.

28. Gillies MT, Coetzee MA. Supplement to the Anophelinae of Africa South of the Sahara 2nd edn. Publ South Afri Inst Med Res. 1987;55:143.

29. Wirtz RA, Ballou WR, Schneider I, Chedid L, Gross MJ, Young JF, et al. Plasmodium falciparum: immunogenicity of circumsporozoite protein constructs produced in Escherichia coli. Exp Parasitol. 1987:63:166-72.

30. Scott J, Brogdon W, Collins F. Identification of single specimens of the Anopheles gambiae complex by PCR. Am J Trop Med Hyg. 1993;49:520-9.

31. Corbel V, Akogbeto M, Damien GB, Djenontin A, Chandre F, Rogier C, et al. Combination of malaria vector control interventions in pyrethroid resistance area in Benin: a cluster randomised controlled trial. Lancet Infect Dis. 2012;12:617-26.

32. Pinder $M$, Jawara $M$, Jarju LB, Salami $K$, Jeffries $D$, Adiamoh $M$, et al. Efficacy of indoor residual spraying with dichlorodiphenyltrichloroethane against malaria in Gambian communities with high usage of long-lasting insecticidal mosquito nets: a cluster-randomised controlled trial. Lancet. 2015:385:1436-46.

33. Ranson H, N'guessan R, Lines J, Moiroux N, Nkuni Z, Corbel V. Pyrethroid resistance in African anopheline mosquitoes: what are the implications for malaria control? Trends Parasitol. 2011;27:91-8.

34. Gatton ML, Chitnis N, Churcher T, Donnelly MJ, Ghani AC, Godfray HC, et al. The importance of mosquito behavioural adaptations to malaria control in Africa. Evolution. 2013;67:1218-30.

35. Oxborough RM. Trends in US President's Malaria Initiative-funded indoor residual spray coverage and insecticide choice in sub-Saharan Africa (2008-2015): urgent need for affordable, long-lasting insecticides. Malar J. 2016;15:146.

36. Smith Gueye C, Newby G, Gosling RD, Whittaker MA, Chandramohan D, Slutsker L, et al. Strategies and approaches to vector control in nine malaria-eliminating countries: a cross-case study analysis. Malar J. 2016;15:2.

37. Masaninga F, Chanda E, Chanda-Kapata P, Hamainza B, Masendu HT, Kamuliwo M, et al. Review of the malaria epidemiology and trends in Zambia. Asian Pac J Trop Biomed. 2013;3:89-94.

38. Pringle G. Malaria in the Pare area of Tanzania. 3. The course of malaria transmission since the suspension of an experimental programme of residual insecticide spraying. Trans R Soc Trop Med Hyg. 1967;61:69-79.

\section{Publisher's Note}

Springer Nature remains neutral with regard to jurisdictional claims in published maps and institutional affiliations. 